This item was submitted to Loughborough's Research Repository by the author.

Items in Figshare are protected by copyright, with all rights reserved, unless otherwise indicated.

\title{
Is the SNARC effect related to the level of mathematics? No systematic relationship observed despite more power, more repetitions, and more direct assessment of arithmetic skill
}

\section{PLEASE CITE THE PUBLISHED VERSION}

https://doi.org/10.1080/17470218.2013.772215

\section{PUBLISHER}

Routledge

\section{VERSION}

AM (Accepted Manuscript)

\section{PUBLISHER STATEMENT}

This is an Accepted Manuscript of an article published by Taylor \& Francis in Quarterly Journal of Experimental Psychology on 01 Oct 2013, available online:

https://doi.org/10.1080/17470218.2013.772215http://www.tandfonline.com/[Article DOI].

\section{LICENCE}

CC BY-NC-ND 4.0

\section{REPOSITORY RECORD}

Cipora, Krzysztof, and Hans-Christoph Nuerk. 2013. "Is the SNARC Effect Related to the Level of Mathematics? No Systematic Relationship Observed Despite More Power, More Repetitions, and More Direct Assessment of Arithmetic Skill". Loughborough University. https://hdl.handle.net/2134/12179055.v1. 
Is the SNARC effect related to the level of mathematics?

No systematic relationship observed despite more power, more repetitions and more direct assessment of arithmetic skill

\section{Krzysztof Cipora}

Institute of Psychology, Jagiellonian University, Kraków, Poland.

\section{Hans-Christoph Nuerk}

Department of Psychology, University of Tuebingen

Knowledge Media Research Center, IWM-KMRC, Tuebingen

Running title: SNARC and arithmetic skills

Cipora, K. \& Nuerk, H.-C. (2013). Is the SNARC effect related to the level of mathematics? No systematic relationship observed despite more power, more repetitions, and more direct assessment of arithmetic skill. The Quarterly Journal of Experimental Psychology, 66, 1974-1991, DOI: 10.1080/17470218.2013.772215

Corresponding author:

Krzysztof Cipora

Institute of Psychology, Jagiellonian University, Kraków.

Al. Mickiewicza 3, 31-120 Kraków, Poland

e-mail: krzysztof.cipora@gmail.com

fax: +48126237699

mobile: +48507946858 


\section{Abstract}

The SNARC (spatial-numerical association of response codes) described that larger numbers are faster responded with the right and smaller numbers with the left. It is held in the literature that arithmetically skilled and nonskilled adults differ in the SNARC. However, the respective data are descriptive and the decisive tests are nonsignificant. Possible reasons for this non-significance could be that previous studies used (i) very small samples, (ii) too few repetitions producing too little power and, consequently, too small reliabilities to reach conventional significance levels for the descriptive skill differences in the SNARC and (iii) general mathematical ability was assessed by the field of study of students, while individual arithmetic skills were not examined.

Therefore we used a much bigger sample, a lot more repetitions and direct assessment of arithmetic skills to explore relation between the SNARC effect and arithmetic skills. Nevertheless, a difference in SNARC effect between arithmetically skilled and nonskilled participants was not obtained. Bayesian analysis showed positive evidence of a true null effect, not just a power problem.

Hence we conclude, the idea that arithmetically skilled and nonskilled participants generally differ in the SNARC effect, is not warranted by our data.

193 words 


\section{Introduction}

The SNARC effect (Spatial Numerical Association of Response Codes, Dehaene, Bossini, \& Giraux, 1993; Fias, Brysbaert, Geypens, \& D’ydewalle, 1996; Nuerk, Wood, \& Willmes, 2005 ) is regarded as evidence for an amodal number-space association (but see Gevers, Santens, Dhooge, Chen, Van den Bossche, Fias, Verguts., 2010). When participants are judging parity of presented numbers in a speeded bimanual setting, they respond faster with the right hand on big magnitude numbers whereas responses to small magnitude numbers are faster on the left hand side. In a recent meta-analysis, it was shown that about $70 \%$ of participants reveal the SNARC effect (Wood, Willmes, Nuerk, \& Fischer, 2008) while $30 \%$ do not show a regular SNARC effect. So, there is certain variability in the SNARC effect that has caught attention of researchers.

Previous results about SNARC effects in skilled and nonskilled participants

Different factors have been proposed to cause this variability in the SNARC effect (e.g., Lindemann, Alipour \& Fischer, 2011, Shaki, Fischer, \& Petrusic, 2009, for recent suggestions and data; Göbel, Shaki, \& Fischer, 2011, for a recent review). Among others, a potential factor influencing the magnitude of the SNARC effect is the level of competence in math. This factor shall be investigated in this study.

Skill as a determinant of SNARC variability was already investigated in the first experiment of the seminal study by Dehaene et al. (1993). Participants studying different subjects were examined (10 presumably mathematically skilled (Science students, later referred as S group) and 10 presumably mathematically nonskilled (Literature students, later referred as L Group)). Participants performed a Parity Judgement Task consisting of 10 repetitions per number in each block and the SNARC effect was analysed. This study has been seen as first evidence for the SNARC effect 
differing between mathematically skilled and nonskilled participants (see e.g. Fischer \& Rottmann, 2005, below; Fischer, Mills, \& Shaki, 2010; Schneider, Grabner, \& Paetsch, 2009 , for children). Therefore a closer look at its results is instructive. The SNARC effect, as it was analysed in this study, showed an overall interaction of side of response with linear contrast for magnitude. There were also interactions of group with parity and magnitude, but no interaction of group with the SNARC effect. Moreover, when those groups were analysed separately, the interaction between side of response and linear magnitude contrast was observed for both the skilled and the nonskilled group. If one takes a closer look at the graphs (e.g. Figure 3), there is a descriptively larger SNARC slope for the L group $(-10.1 \mathrm{~ms})$ than the $S$ group $(-4.1 \mathrm{~ms})$. So, we might want to interpret this as a hint that the L group produced a larger SNARC slope than the S group, but, as mentioned above, there is no significant SNARC interaction with group in the inference-statistical analyses. Moreover, no direct comparison between the groups regarding the SNARC effect is reported (non-significant results were not reported on in detail in the paper).

It is important to note that significant interactions of group with numerical variables were observed. For instance, the interaction of group with parity was significant which was driven by a significant odd effect (i.e., generally faster responses to even numbers compared to odd numbers; Hines, 1990) in the literary group but not in the science group. Interestingly, the odd effect has been linked to a linguistic concept, namely linguistic markedness: In most languages, in a pair of complementary adjectives, only one; linguistically unmarked - can be negated by adding relevant prefix efficient / inefficient. Besides this negation criterion, unmarked forms may be detected by distributive markedness (e.g. larger frequency) or by semantic markedness (e.g. being 
the default form in questions (e.g., how old are you?; Lyons, 1969; Nuerk, Iversen, \& Willmes, 2004). Unmarked concepts are commonly responded faster to (e.g. Hines, 1990). In most languages "even" is considered unmarked. So, assuming that the students in literary studies are more concerned with linguistic contents, it may not be so surprising that they exhibit a larger linguistic markedness effect, namely, in this case a larger odd effect.

To summarize the Dehaene et al. results about the difference in SNARC between mathematically skilled and nonskilled participants, the graphs hint that the SNARC effect for nonskilled participants may be larger, but the respective interaction is not significant. As mentioned above, a direct group comparison was not reported and the SNARC effect was apparently observed in both groups. Nevertheless, the study is seen as evidence for relationship between the SNARC effect and skill until today (see e.g. Fischer \& Rottmann, 2005, below; Fischer et al., 2010; Schneider et al., 2009).

The difference in the magnitude of the SNARC between mathematically skilled and nonskilled persons was further investigated by Fischer and Rottmann (2005). Again 10 participants of each group were examined and each number was presented 9 times in a block. Fischer and Rottmann described their results as follows: "Replicating Dehaene et al.'s (1993) finding, there was a weaker SNARC effect in the skilled group. However, this difference failed to reach significance here, $t(18)=1.12, p=0.28 . "$ In our opinion, this description can be slightly misleading. As described above in detail, the observed SNARC effect in Dehaene's study was not significantly weaker in the skilled group. Rather, both studies found a descriptive tendency for a weaker SNARC 
effect in the skilled group, but this group difference failed to reach significance in both studies.

Based on this review, we are left with the question, whether the descriptive tendency in both studies may, nevertheless, be valid since there seem to be good reasons as to why a significant result has not been found. These possible reasons for a null effect are discussed in the next section.

\section{Reasons for the observed null effects}

The first reason for the non-significance of these differences could simply be a lack of power. In both studies mentioned above examined groups were indeed relatively small. Additionally the number of repetitions in the task may not have had sufficient power to detect reliable group differences in the SNARC. Recent simulations indicated that the power to detect significant group differences as regards the SNARC depend heavily on the number of repetitions (Cipora \& Wood, 2012). Small number of repetitions per single conditions shortens the task length. Shorter tasks or tests and can lead to decrease in reliability of that test result, in our case the estimation of the SNARC effect. The consequence of such lower reliability is a lower chance of finding between-group differences of the SNARC effect. Therefore we used not only a much larger number of participants, but also more repetitions in the SNARC task than in previous studies to increase measurement reliability.

Finally, one might argue that participants were not grouped according to their actual mathematical competence. For instance, some students studying non-math subjects might be fluent and skilled in math as well. This might be especially detrimental for the study because the groups were relatively small. To deal with this point, in addition to selecting a group of students with a math-related and with a math-unrelated subject, a 
mental calculation task was used to measure the actual individual level of arithmetic skills (i.e. participants were to verify accuracy of equations presented on the computer screen).

\section{Alternative sources of variability between groups}

It is also possible that not the mental number line differs between skilled and unskilled participants, but that differences in the SNARC effect are produced by confounded sources of variability. One such source of SNARC effect variability was pointed out by Gevers, Verguts, Reynvoet, Caessens and Fias (2006). Participants who perform parity judgment faster display a smaller SNARC effect than those who react slower. This tendency was also observed across studies that were meta-analysed by Wood et al. (2008). So, the nonskilled participants may just have been slower and therefore exhibited a larger SNARC effect. To rule out that any group differences are due to overall slowing or speeding up, a measure of overall response speed should be included in a SNARC analysis as a covariate. We thus used a simple reaction time task in this study (i.e. participants were asked to respond as soon as they notices simple visual stimuli) to obtain a task-independent measure of overall processing speed. Additionally, we included overall RT in the Parity Judgment Task itself as a covariate.

\section{Hypothesis}

With these four improvements, (i) a lot more participants, (ii) twice as much repetitions (iii) independent assessment and covariate inclusion of processing speed and (iv) additional independent measure of arithmetic skills, we should be able to detect differences in the SNARC effect between mathematically nonskilled and skilled participants if they existed and just failed to reach significance in previous studies. 
Moreover, we should be able to rule out alternative accounts such that larger SNARC effect differences between groups are based on pure speed differences between groups. 


\section{Method}

\section{Participants}

71 (49 female) participants took part in the study for course credit1. 53 of them (44F) were studying subjects in which Math competence is not crucial (e.g. psychology, literature, law; "Non-Math" group). The remaining 18 (5 F) were studying Math-related subjects (e.g. Math, computer science, engineering; "Math" group). The mean age was 21.8 years $(\mathrm{SD}=1.9$, range $19-28)$.

\section{Materials}

The study consisted of three computerized tasks (all run on DMDX program, Forster \& Forster, 2003). In all tasks grey background (\#21021010 in RGB notation) was used to avoid very sharp contrast with the stimuli. Stimuli were presented with black Arial font. Parity Judgment Task: Participants were to assess whether a centrally presented number was odd or even. Eight numbers were used (1, 2, 3, 4, 6, 7, 8, 9), font size 30 . Each number was preceded by an eye fixation point (300ms). A number was presented until the participant responded or for $2 \mathrm{~s}$. After $500 \mathrm{~ms}$ the next trial started. The task consisted of two blocks differing in assignment of the response keys, which were the keys P and Q on a standard PC keyboard. The order of blocks was counterbalanced across participants. Each block was preceded by a training session of 8 trials in which feedback was provided. In experimental blocks each number was presented 20 times 2 , so that the task consisted of 320 trials in total.

Simple Reaction Time: The participant's task was to react as soon as she or he noticed an asterisk $\left({ }^{*}\right)$, font size 34 , appearing centrally on the screen. Each target was presented until response or for 1s and was followed by an ISI varying between 800 and $1300 \mathrm{~ms}$. The task consisted of 300 trials preceded by training (10 trials). Standard computer USB mouse was used to collect responses. 
Equation Verification Task: Participants were to verify whether the equation presented on the computer screen (font size 30) was correct or not by pressing a key. There were four subsets of problems (1) Addition and subtraction of two-digit numbers (e.g. 95-13=82 - correct), (2) One-digit by one-digit/two-digit number multiplication (e.g. 6*43=248 - incorrect), (3) Two-digit by one-digit/two digit number division (e.g. 55/11=6 - incorrect), (4) Order of operations and parentheses (e.g. $21+5 * 4=41-$ correct). All problems are listed in Appendix I. Each subset consisted of 20 equations (50\% correct) so that the task consisted of 80 trials in total and was preceded by a short training (4 trials) during which feedback was provided. Order of trials within task (i.e. across subsets) was randomized.

Procedure:

After signing an informed consent, participants were seated in front of the computers and presented with 3 paper and pencil questionnaires: 2 measuring anxiety (two parts of STAI State -Trait Anxiety Inventory, Spielberger, Gorsuch, Lushene, 1970) and one measuring tension (LPT - Polish version of Activation-Deactivation Adjective Check List; Thayer, 1986)з. Then the participants were to perform 3 computerized tasks (in order as reported): Parity Judgment, Simple Reaction Time and Equation Verification. The session lasted about 35 minutes. 


\section{Results}

Data preparation

For Parity Judgment only correct responses were analysed. In Parity Judgment as well as in Simple Reaction Time outlier responses were removed sequentially. At first unnaturally short responses were removed $(<80 \mathrm{~ms}$ for Simple RT and $<150 \mathrm{~ms}$ for Parity Judgment). Then average response latencies as well as SD in response latencies were calculated. Reactions deviating by more than \pm 3 SD from average were removed. Then average RT and SDs were calculated again and the whole procedure was repeated until there were no more changes in averages within every participant. For Equation Verification no trimming was used.

\section{Parity Judgment}

The average error rate was 5.6\% (SD=3.6\%). There was no speed-accuracy trade-off $(r=0.03 ; p=0.831)$. Speed-accuracy trade-off was also not found in groups when analysed separately: For the "Math" group the correlation between average RT and number of errors was $-0.17(p=0.502)$, for the "Non-Math" group it was $0.03(p=0.802)$, and these correlations did not differ significantly $(p=0.490) .90 \%$ of the data was included in the analysis (i.e. errors and outliers removed). Further results are reported on the data after trimming.

The mean reaction time was $528 \mathrm{~ms}(\mathrm{SD}=122 \mathrm{~ms}$ ). Reaction times differed between groups. The "Math" group performed the task faster than the "Non-Math" group $(\mathrm{M}=499, \mathrm{SD}=118$; and $\mathrm{M}=538, \mathrm{SD}=61 \mathrm{~ms}$ respectively). Variances of overall RT were unequal (Leven's test significant $\mathrm{p}=0.002$ ), therefore an adjusted t-test was used. The difference was not significant $\mathrm{t}_{20.22}=1.34 ; \mathrm{p}=0.194$. 
For estimating the magnitude of the SNARC effect, the analysis proposed by Fias at al. (1996) was conducted. Reaction times were averaged for each hand / each number for each participant. Then dRT's were calculated (RT right hand - RT left hand) for each participant and each number. A positive value of dRT indicates a faster left hand response compared to the right hand response. The dRTs were then regressed on numerical magnitude using linear regression for each participant separately. The unstandardized value of the regression slope can be interpreted as a measure of the SNARC effect (Slope) - the more negative the value, the stronger the SNARC effect. The mean slope was $-4.78(\mathrm{SD}=7.18)$. The presence of a SNARC effect was examined by testing slopes against 0 using a one-sample t-test. The SNARC effect was highly significant t70=-5.61; $\mathrm{p}<0.001$. Slopes for the "Math" group were descriptively smaller than for the "Non-Math" group (M=-3.96, $\mathrm{SD}=5.19$ and $\mathrm{M}=-5.07, \mathrm{SD}=7.59$ respectively). Nevertheless, the difference was far from significance ( $\mathrm{t} 69=0.56 ; \mathrm{p}=0.575)$. Levene's test $(F=0.473 ; p=0.494)$ showed no indication of unequal variances for SNARC slopes therefore unadjusted t-test was used for comparison.

\section{Simple reaction time}

$89 \%$ of reactions met the trimming criteria. Further results are reported on the data after trimming. The average reaction time was $243 \mathrm{~ms}(\mathrm{SD}=61 \mathrm{~ms})$. The "Math" group was faster than the "Non-Math" group (M=226 ms; SD=49 and M=247ms; SD=29 respectively). Variances were unequal (Leven's test significant $\mathrm{p}=0.037$ ), therefore an adjusted t-test was used. The difference was not significant $t 20.92=1.67 ; p=0.109$.

\section{Equation verification}


The mean accuracy was $66.7(\mathrm{SD}=8.1)$. The "Math" group performed better than the "Non-Math" group (M=73.1, $\mathrm{SD}=3.2$ and $\mathrm{M}=64.6, \mathrm{SD}=8.1$ respectively). This difference was highly significant t67.36=-6.30; $<<0.001$. Variances were unequal (Leven's test significant $\mathrm{p}<0.001$ ), therefore an adjusted t-test was used.

The average time of equation solving (for accurate trials) was 3678ms (SD=920). The "Math" group performed faster $(M=3312 \mathrm{~ms}, \mathrm{SD}=873$ versus $\mathrm{M}=3803 \mathrm{~ms}, \mathrm{SD}=910)$. This difference was significant $\mathrm{t}_{69}=2.0 ; \mathrm{p}=0.05$. Nevertheless, this result must be taken with caution because only correct responses were taken into account, so if participant A solved only the simplest trials, he or she could have been faster than participant B who solved difficult trials as well, which naturally take more time.

We also took a more detailed look at the four subsets of the Equation Verification task (addition/subtraction, multiplication, division, order of operation; tasks and stimuli see Appendix I). Average accuracy was highly correlated across subsets (see Table 1). Measures of reliability (split-half, Cronbach alpha) were lower, sometimes much lower for the single subsets $(\mathrm{r} 1 / 2=.48$, Cronbach alpha $=.40$, for subset 2 : multiplication) Further characteristics of the task (different measures of homogeneity and consistency, and relations to SNARC) are reported in detail in Appendix III.

(Table 1 about here)

\section{Reliability of measures}

To examine whether eventual null group differences and null correlations were due to low reliability of the SNARC effect, reliability of all measures, the SNARC slopes and the predictors was computed. Computations were based on split-half reliabilities using the odd-even method to control for systematic influences of practice or tiring within task as follows for the different tasks. For Simple Reaction Time, reaction times were calculated 
separately for odd and even trials. For Equation Verification, trials were half-split within each subset and global reliability is reported (as mentioned above, further task characteristics, including reliability for each subset, are provided in Appendix III). In Parity Judgment trials were odd-even (due to order of appearance) half-split. Based on that division, two slopes were calculated separately for each participant (based on 10 repetitions of each number per condition). The same split was used for calculating average reaction times as well as variability in reaction times in Parity Judgment. This split-half correlations were Spearman-Brown corrected - as is common for split-half correlations because the reliability of the full test / task shall be estimated and not only of the half test/task. All reliability results are presented in Table 2. The reliability of SNARC slopes was rather high in our adult sample $\left(\mathrm{r}_{1 / 2}^{1 / 2}=.698\right)$ probably because of the increased numbers of repetition we used in this study. The reliability of all other predictors was above .90 . Thus, these results suggest that null correlations of skill with SNARC slopes or null group differences cannot be due to a general lack of reliability of these measures.

(Table 2 about here)

Factors influencing the magnitude of the SNARC effect

In order to provide a full overview of our results we calculated zero order correlations between the SNARC slopes and all other variables potentially influencing the SNARC effect (see Table 3). Only mean RT and SD of mean RT in parity judgment task correlated significantly with the SNARC effect.

(Table 3 about here) 
Additionally, we conducted multiple regressions to find unique variance relations between the SNARC slope and predictor variables when statistically controlling for influences of other remaining predictors. The predictors were as follows: (1) RT in Parity Judgment Task, (2) Simple RT, (3) Equation verification accuracy, (4) Equation Verification RT, (5) Group. The regression was significant F5. $70=2.44 ; \mathrm{p}=0.044$ and explained $15.8 \%$ of the variance (R2adj $=0.093), S E=6.84$. The only significant predictor was RT in Parity Judgment itself. Participants who were faster in Parity Judgment revealed a smaller SNARC than those who were relatively slow (see Table 4).

(Table 4 about here)

Even when Equation Verification Accuracy and Equation Verification RT were entered as first predictors they did not explain any variance of the SNARC slope $(p=.18$ and .14 respectively). When the other predictors were added subsequently only parity mean RT reached significance $(p=.007)$. In sum the results were not substantially different from regression analysis in which all parameters were entered together.

Typically, in cognitive experiments means are analysed to distinguish between different subjects and the ease, with which they performed the task at hand and have time to also activate task-irrelevant representations (in this case magnitude). However, in other diagnostic tasks such as RT tests for attention, standard deviations are also analysed as an alternative measure to examine whether an individual can solve the task consistently and with ease or whether influences of other representations and processes (such as magnitude) do influence the task in an inconsistent manner in some trials. Therefore, 
we used both, means and standard deviations in different regressions (due to high collinearity of mean RT and SD of RT).

For the regression with SD measures the predictors were: (1) SD of RT in parity judgment task; (2) SD of Simple RT, (3) Equation verification accuracy, (4) SD of RT in Equation Verification, (5) Group. This regression explained 16\% of the variance (R2adj=0.097), $S E=6.83$ and was significant $\left(F_{4}, 70=2.50 ; p=0.039\right) . S D$ of $R T$ in Parity Judgment was the only significant predictor $(\mathrm{p}=0.002)$.

As suggested by the reviewers, we also took a more detailed look in multiple correlations of the single subsets of the verification task and the questionnaires with the SNARC effect. When adjusting for multiple comparisons, none of these correlations was significant. Without adjustments for multiple comparisons, one single correlation (subset 3: division) reached conventional significance levels (see Appendix III). However, while this correlation was (without adjustment) larger than zero, it was not significantly different from the correlations for the other subsets of the Equation Verification Task (addition/subtraction, multiplication, order of operations, see Appendix I). A specific relationship of SNARC with division skill is therefore not warranted by the data.

In sum, group or direct global measures of arithmetic skill were not even close to significantly predicting SNARC slopes. Multiple correlations with subsets of the Equation Verification Task and the Questionnaires did not reach significance when corrections for multiple comparisons were applied. As can be seen in Figure 1, panels AD, neither the correlations nor the null results are due to unequal variances or outliers.

(Figure 1 about here) 
Participants revealing negative SNARC slopes

One could have argued that average null SNARC effects between groups have been due to single few participants in one group with unusual, highly positive or highly negative SNARC effects. Therefore, we also used another way of examining group differences by looking at the proportion of participants revealing negative SNARC slopes (i.e. SNARC effect) in the two groups. As mentioned in the introduction, proportion of participants revealing negative SNARC slopes is usually constant across studies (c.a. 70\%). On the other hand, as it was found by Fischer (2008), in groups revealing atypical SNARC pattern, apart from difference in slopes, higher proportion of participants revealing reverse SNARC can be seen. Figure 2 depicts the proportion of participants revealing negative SNARC slopes. This proportion did not differ across groups either: $\operatorname{chi} 2(1)=0.002, p=0.966$

(Figure 2 about here)

Bayesian analysis

Keeping in mind controversies regarding confirmation of null hypothesis using traditional statistical inference, Bayesian method was used. The method described in detail by Masson (2011) enables calculating graded evidence for null hypothesis (i.e. no difference between groups) and alternative hypothesis (i.e. difference between groups)4. In the analysis, sum of squares and number of observations from ordinal ANOVA are used to calculate Bayesian factors, which then can be used to calculate posterior probabilities. The analysis was conduced both for study subject and arithmetic skill comparisons of the SNARC slopes. 
Study subject: The comparison between the "Math" and "Non-Math" groups was made with one factor ANOVA (analogous to t-test comparison reported above, so the actual p value for this comparison is the same as it was in the $t$ test reported i.e. $p=0.575$ ). Bayesian analysis revealed that, based on our data, the posterior probability of null hypothesis was 0.88 (the same probability of alternative hypothesis was complimentary i.e. 0.12 ). This is rated as positive evidence for the null hypothesis applying by the criteria suggested by Masson (see also Raftery, 1995) and it is even slightly above the ratio of $84,9 \%$ (as compared to $15.1 \%$ for the null hypothesis there) of the very experiment, which he gave himself as a positive example for the application of Bayesian statistics.

Arithmetic skill: The scores in Equation Verification task were median split and the slopes were compared across groups. $\mathrm{F}_{1,69}=0.60 ; \mathrm{p}=0.443$. Mean slope in the group above median was $-4.13(\mathrm{SD}=7.25)$ and for the group below median the mean slope was $-5.45(\mathrm{SD}=7.16)$. Most importantly, again the Bayesian analysis revealed that the evidence in favour of null hypothesis was 0.86 , and the complimentary evidence for alternative hypothesis was 0.14 . These values can be interpreted as positive evidence in favour of no difference between groups as well.

We also computed the same Bayesian analyses for the single subsets. Because of their lower reliability, subset scores are less conclusive. In particular, the Bayesian analysis for subset 3: division, for which a significant correlation with SNARC was obtained without adjustment, revealed an even slightly higher probability of the null hypothesis $(p=.55)$ than for the alternative hypotheses $(p=.45)$. Thus, there is no evidence from the Bayesian analysis that this single subset correlation getting significant only without adjustment is pointing to a true SNARC difference between skilled and unskilled division performers. 


\section{Discussion}

It has been claimed that mathematically skilled and nonskilled participants differ in their SNARC effects (Fischer \& Rottmann, 2005; Fischer et al., 2010). However, in such studies only descriptive differences were obtained: Nonskilled participants showed descriptively larger SNARC slopes, but these differences always failed to reach conventional significance levels (e.g., Dehaene et al., 1993; Fischer \& Rottmann, 2005)..

Failure to reach significance in those studies may have been due to at least four sources:

(i) Too few participants

(ii) Too few repetitions per data point

(iii) Mathematical skill was determined on the basis of study field and not directly assessed.

(iv) Differences in overall RT, which can lead to a larger SNARC effect, were not directly assessed.

In our present study, we dealt with all these issues. We used a larger sample than almost any other SNARC study (see e.g. meta-analysis by Wood et al., 2008, for information about previous SNARC studies). We also used 20 repetitions per data point to enhance reliability of the SNARC effect (see Cipora \& Wood, 2012, for simulations) while Dehaene et al. (1993) and Fischer and Rottmann (2005) employed only 9 or 10 repetitions. Moreover, the level of arithmetic skill was directly assessed by behavioural performance and not only by field of study. Finally, we assessed the general processing speed of participants in an independent task (Simple RT) because it is known that slower RTs lead to larger SNARC effects (Gevers et al., 2006). In this way, we could test 
whether eventual group differences in the SNARC effect are rather due to differences in processing speed between participants groups than to arithmetic skill differences. In sum, we tried hard to create an experimental setting in which we could finally show a significant difference in the SNARC effect between mathematically skilled and nonskilled participants.

Issues of power and particular analyses methods

We also took great care in numerous analyses to ensure that our results were not due to a particular analysis methods or poor power. We correlated the SNARC slopes both with math group and arithmetic skill. We computed these correlations for raw and standardized SNARC slopes to ensure that null effects are not due to individual differences in SNARC slope variance. Moreover, we used regression analyses to search for unique variance of different predictors. In addition to those parametrical analyses, we looked at the proportion of possible SNARC slopes for mathematically skilled and unskilled participants to ensure that null effects are not due to one of few extreme outlier slopes.

We also addressed the power issue by various analyses. We computed reliabilities of all correlated tests and corrected correlations for attenuation. Moreover, we examined correlations of the SNARC effect to other measures (such as overall RT and standard deviations). These correlations were significant and ensured discriminant validity, i.e., that the SNARC effects are not just uncorrelated to anything else tested in our study). Finally, we employed Bayesian statistics to estimate the likelihood of correctness of the null and alternative hypotheses. 
In sum, also motivated by reviewers' requests, we ran and report more extensive statistical analyses than in any study before on the relation between SNARC slope and skill (see Appendices for additional details).

No systematic relationship between SNARC and arithmetic skill

Despite all these experimental and statistical efforts, the results were disillusioning with regard to the hypothesis that SNARC slope and arithmetic skill are related. The influence of any skill-related manipulation failed to reach significance by far $(p=.58$, standard group comparison, $\mathrm{p}=.54$ influence of arithmetic skill predictor in regression, $\mathrm{p}=.93$ for group predictor in regression). Neither skill group (as indexed by field of study) nor individual skill (as indexed by an independent arithmetic skill test) influenced the SNARC effect systematically. We even analysed the influence of standard deviations or employed effect sizes for each person rather than standard SNARC slopes (not reported in detail), but there was no influence of skill group or individual skill in these additional analyses. Finally, the proportion of participants revealing negative SNARC slopes was around $70 \%$ and is in line with the meta-analyses reported by Wood et al. (2008). This proportion did not differ between the "Math" and the "Non-Math" group ( $p=.97$ for chi2-test of distribution of SNARC slopes). So, the null differences between the groups cannot be due to one or a few outliers. Importantly, these null effects are not due to a lack of reliability of the measures we used. All predictors we used had very good reliabilities (see Table 2), and even the reliability of SNARC slopes was satisfactory $\left(\mathrm{r}_{1 / 2}=.698\right)$, probably because we used much more repetitions in the SNARC task than any such study before. Therefore, the failure to find any relation between the SNARC effect and skill cannot be due to a lack of reliability in our study. 
We agree that the evidence emerging from traditional statistical inference methods can be challenged as far as confirming null hypothesis (i.e. no difference between groups). However, our conclusion is supported by the results from the Bayesian analyses. They showed positive evidence in favour of no difference between skilled and nonskilled groups, for both, study subject and arithmetic skill. Again, the same results were obtained when raw and standardized slopes were analysed (not reported here in detail).

Lack of group difference can also not be accounted for by differing speed-accuracy trade-offs (i.e. skilled group devotes speed to be more correct whereas nonskilled group devotes accuracy to be faster or reverse). Speed-accuracy trade-off was not observed in the whole sample taken together or in groups when analysed separately.

All in all, even with more participants than ever before, more repetitions, direct and independent assessment of arithmetic skill and overall response speed, with much more extensive analysis of reliability, power and speed-accuracy trade-offs than in any such study before, there is no indication of any difference in the SNARC effect between skilled and nonskilled participants. This is clearly at odds with the claims in the literature concerned with adults (Fischer \& Rottmann, 2005; Fischer et al., 2010). However, our results for adults seem to be in line with recent children data presented by Schneider and colleagues (2009). In school children (5th and 6th grade), they observed no relation between magnitude of the SNARC effect and mathematical achievement. Taken together, in recent studies in children (Schneider et al., 2009) and adults (this study) suggest that there is no systematic relation between SNARC and arithmetic skill at any point of development. 
It is important to note that our study was not generally producing null effects. We observed highly significant SNARC effects (which is not surprising given the large $\mathrm{N}$ and the high number of repetitions) and significant influences of other factors on the SNARC: Regressions revealed that participants who respond slower in the parity judgement task seemed to have a stronger SNARC effect. This is in line with the results by Gevers et al. (2006) and meta-analysis data shown by Wood et al. (2008). Moreover, the magnitude of the SNARC effect in our study also depends on the SD of RT in Parity Judgment, not RT per se. Participants who were more variable in their reaction latencies revealed a larger SNARC effect as well. However, these influences did not modulate any group differences (or lack thereof) in the SNARC effect.

Finally, we can expand the conclusion of Gevers et al. (2006), in saying that the magnitude of the SNARC effect is related not only to overall response latencies but also and more so to stability in response latencies in Parity Judgments.

To summarize, not only stable SNARC effects, but also significant influences on the SNARC effect from other variables consistent with the previous literature were observed. However, none of these influences altered the lack of differences in the SNARC effect between skilled and nonskilled participants.

Limitations of the study: Mathematical competence vs. the assessment of arithmetic skills In previous studies, the field of study was used as an index of general mathematical ability. These previous studies did not use any individual assessment of mathematical or arithmetic skills. For this study, an Equation Verification Task was developed to index arithmetic skills. In doing so, we are in the tradition of numerical cognition research, which usually used more complex arithmetic tasks to assess more complex 
numerical understanding (see e.g., Nuerk, Moeller, Klein, Willmes, \& Fischer, 2011 for a review including complex arithmetic in the multi-digit number range).

However, it has to be acknowledged that arithmetic skill is only a part of general mathematical competence, which may additionally include understanding and developing mathematical proofs, theorems and models (e.g,, Pesenti, 2004), efficiency of the number sense (e.g. manipulating approximate quantities; Dehaene, 2001) as well as many other capabilities. Although the Equation Verification Task was reliable and valid in that it differentiated arithmetically skilled and nonskilled participants in both on accuracy and speed of solving trials, this task is not a psychometrical tool for measuring general math competence. Therefore, an established psychometric test measuring broader range of math competence including proofs and theorems would be desirable as it remains possible that students with different SNARC effects may still differ in such more mathematical tasks or tests.

In fact, there is a single result in our study, which may suggest that future studies should examine separate arithmetic skills in more fine-grain detail. When analysing multiple SNARC correlations with questionnaire measures and Equation Verification subsets, we observed one significant correlation of the SNARC effect with division performance, when we did not adjust for multiple comparisons. However, this correlation did neither reach significance when multiple comparisons are corrected, nor did it differ significantly from the SNARC correlations with the other subsets. Additionally, Bayesian analysis suggested that the likelihood of the alternative hypothesis being true (good division performers showing smaller SNARC slopes) is only $\mathrm{p}=.45$ while the likelihood for the null hypotheses is $p=.55$. So, our data do not warrant a strong conclusion, that we are really looking at a single specific exception from the general null correlation and not only at some random significance because of multiple correlations. Nevertheless, 
this deserves further investigation in the future, if one particular arithmetic skill is closer related to the SNARC effect than other skills.

In sum, the null effects observed here hold only for the arithmetic skills assessed and the mathematical capabilities associated with the selected subject fields in this study. However, this does not preclude that SNARC differences may be related to other mathematical or number sense domains or even other psychometric tests of arithmetic skills.

Diverging results in the literature: On the need for more fine-grain analyses In the introduction, we have outlined that generally it has been claimed that the SNARC is related to math competence. However, in the literature diverging results are now coming up. In a colour discrimination task examining 40 participants (Bull, Cleland \& Mitchell, 2012, Experiment 2) there was no relation between math competence (as measured with Numerical operations form the Wechsler Individual Achievement Test WIAT II-UK) and magnitude of the SNARC effect. Reported correlation between slopes and the test score was -0.07 . Examining relations between magnitude of SNARC and math competence was not the main goal of the experiment and these relations were not investigated in more detail there. However, it is important that the SNARC effect was not measured by typical parity judgment but modified version of colour discrimination task, which is not considered semantic. This lack of semantic processing may explain the null correlation between SNARC effect and arithmetic performance in Bull et al. (2012), but not in our study.

On the other hand, diverging results to this study and ours were reported by Hoffmann, Mussolin, and Schiltz (2012). They seem to find relationships between the SNARC and arithmetic skills. There are some differences between their and our study, which 
deserve attention in future studies, such as the inclusion of zero in the experiment of Hoffmann et al. (see Nuerk, et al., 2004, for data showing a special role of zero), inclusion of a low skill group, procedural differences in SNARC and evaluation tasks, and, possibly, cultural differences (cf. Göbel, at al., 2011) as Hoffmann et al.'s study was conducted in a trilingual culture (Luxembourg).

These diverging results may suggest that the nature of the relationship between SNARC and arithmetic skills is not yet fully resolved. Rather, the observation of such a relationship may depend on particular cultures, particular stimuli, particular skill groups, particular SNARC procedures, task designs and computations, particular evaluation tasks. In future studies, these relations should be examined in more finegrain detail with reference to above factors In doing so, we wish to advocate that null effects like the ones obtained here should be published to constrain and foster psychological theory building about the SNARC effect. We do currently see very intense debate on general replicability of psychological studies results (see Special Issue in Perspectives in Psychological Science: Special Section on Replicability in Psychological Science: A Crisis of Confidence?; 2012). Several researchers are deeply concerned that in psychology mostly positive results are published (see e.g. Ferguson \& Heene, 2012). This general tendency leads to literal unfalsifiability of psychological theories because reporting null results is strongly discouraged (see also Bakker, van Dijk \& Wicherts, 2012). However, to understand the generality as well as the fine-grain details about the relationship of the SNARC effect and math competence /arithmetic skills, it is in our view necessary to publish the positive results as well as the null effects to get the full picture.

Implications on the current debate on the sources of the SNARC effect 
The current study was not designed to examine whether the foundation of the SNARC effect is more visuo-spatial or more verbal in nature (Fias, van Dijck, \& Gevers, 2011; Gevers et al., 2010; van Dijck \& Fias, 2011). However, let us assume with Gevers et al. (2010) that the SNARC in a parity judgment task indexes less spatial-numerical representations and rather more verbal-linguistic (or verbal working memory) capacities. Under this assumption, it is not so surprising that mathematically skilled and mathematically nonskilled participants do not differ in the SNARC effect (unless someone postulates that verbal working memory differs greatly between students studying math-related or math-unrelated subjects).

However, the current study also does not rule out a mainly spatial-numerical account of the SNARC effect. After all, neglect patients fail to show particular deficits in the SNARC effect in a parity judgement task although their mathematical abilities can be severely impaired (Priftis, Zorzi, Meneghello, Marenzi, \& Umilta, 2006; Zorzi, Bonato, Treccani, Scalambrin, Marenzi, \& Priftis). Skilled participants may still have a better number line than nonskilled participants, but their automatic (possibly implicit) access to this number line may not differ from the access of nonskilled participants.

\section{Conclusions}

The SNARC effect has been repeatedly suggested to be an index of arithmetic abilities in literature. Descriptive tendencies supporting such claims have been published, however, conventional significance levels were not reached. The current study with (i) the largest number of participants, (ii) the most repetitions per trial of any study examining the SNARC effect for mathematically skilled and nonskilled participants, (iii) an independent assessment of arithmetic skills and (iv) overall RT failed to give any systematic indication that the SNARC effect indeed differs between arithmetically 
skilled and nonskilled participants. We conclude that the data do not support the notion that the SNARC generally differentiates between skilled and nonskilled participants as has been suggested by previous literature. 


\section{References}

Bakker, M., van Dijk, A. \& Wicherts, J. M. (2012). The rules of the game called Psychological Science. Perspectives on Psychological Science 7, 543-554. doi:10.1177/1745691612459060

Bull, R., Cleland, A. A., \& Mitchell, T. (2012, April 30). Sex differences in the spatial representation of number. Journal of Experimental Psychology: General. Advance online publication. doi: $10.1037 / \mathrm{a} 0028387$

Cipora, K., \& Wood, G. (2012). Optimal power to detect (between group differences) in SNARC - Monte Carlo study. Poster presented on XXXth European Workshop on Cognitive Neuropsychology, Bressanone, Italy.

Dehaene, S. (2001). Precis of the number sense. Mind \& Language, 16, 16-36. doi: 10.1111/1468-0017.00154

Dehaene, S., Bossini, S., \& Giraux, P. (1999). The mental representation of parity and number magnitude. Journal of Experimental Psychology: General 122, 371-396. doi: 10.1037/0096-3445.122.3.371

Ferguson, C. J. \& Heene, M. (2012). A vast graveyard of undead theories: publication bias and psychological science's aversion to the null. Perspectives on Psychological Science 7, 555-561. doi:10.1177/1745691612459059

Fias, W., Brysbaert, M., Geypens, F., \& D’ydewalle, G. (1996). The importance of magnitude information in numerical processing: Evidence from the SNARC effect. Mathematical Cognition, 2, 95-110. doi: 10.1080/135467996387552

Fias., W., van Dijck, J. -P., \& Gevers, W. (2011). How number is associated with space? The role of working memory. In: S. Dehaene, \& E. Brannon (Eds.), Space, Time and Number in the Brain: Searching for the Foundations of Mathematical Thought (pp. 133-148). London, UK: Elsevier. doi: 10.1016/B978-0-12-385948-8.00010-4 
Fischer, M. H. (2008). Finger counting habits modulate spatial-numerical associations. Cortex, 44, 386-92. doi:10.1016/j.cortex.2007.08.004

Fischer, M. H., Mills, R. A, \& Shaki, S. (2010). How to cook a SNARC: number placement in text rapidly changes spatial-numerical associations. Brain and Cognition, 72, 333-336. doi: 10.1016/j.bandc.2009.10.010

Fischer. M. H., \& Rottmann. J. (2005). Do negative numbers have a place on the mental number line? Psychology Science. 47, 22 - 32.

Forster, K. I., \& Forster, J. C. (2003). DMDX: A windows display program with millisecond accuracy. Behavior Research Methods, Instruments, \& Computers, 35, 116124. doi: 10.3758/BF03195503

Gevers, W., Verguts, T., Reynvoet, B., Caessens, B., \& Fias, W. (2006). Numbers and space: A computational model of the SNARC effect. Journal of Experimental Psychology. Human Perception and Performance, 32, 32-44. doi: 10.1037/00961523.32.1.32

Gevers, W., Santens, S., Dhooge, E., Chen, Q., Van den Bossche, L., Fias, W., \& Verguts, T. (2010). Verbal-spatial and visuospatial coding of number-space interactions. Journal of Experimental Psychology: General, 139, 180-190. doi: 10.1037/a0017688

Göbel, S. M., Shaki, S., \& Fischer, M. H. (2011). The cultural number line: A review of cultural and linguistic influences on the development of number processing. Journal of Cross-Cultural Psychology, 42, 543-565. doi: 10.1177/0022022111406251

Hines, T. M. (1990). An odd effect: Lengthened reaction times for judgments about odd digits. Memory \& Cognition, 18, 40-46. doi: 10.3758/BF03202644

Hoffmann, D., Mussolin, C., \& Schiltz, C. (2012). The interaction between number and space processing and math achievement in adults. Cognitive Processing, 13, 1, supplement 1, 60 . 
Lindemann, O., Alipour, A., \& Fischer, M. H. (2011). Finger counting habits in Middle-Eastern and Western individuals: An online survey. Journal of Cross-Cultural Psychology, 42, 566-578. doi:10.1177/0022022111406254

Lyons, J. (1969). Semantics. Cambridge: Cambridge University Press.

Masson, M. E. J. (2011). A tutorial on a practical Bayesian alternative to nullhypothesis testing. Behaviour Resesearch Methods, 43, 679-690. doi: 10.3758/s13428010-0049-5

Nuerk, H. -C., Iversen, W. \& Willmes, K. (2004). Notational modulation of the SNARC and the MARC (linguistic markedness association of response codes) effect. Quarterly Journal of Experimental Psychology: A, 57, 835-863. doi:10.1080/02724980343000512

Nuerk, H. -C., Moeller, K., Klein, E., Willmes, K., \& Fischer, M. H. (2011). Extending the Mental Number Line - a review of multi-digit number processing. Journal of Psychology, 219, 3-22. doi:10.1027/2151-2604/a000040

Nuerk, H. -C., Wood, G., \& Willmes, K. (2005). The universal SNARC effect. The association between number magnitude and space is amodal. Experimental Psychology 52, 187-194. doi: 10.1027/1618-3169.52.3.187

Pesenti, M. (2005). Calculation abilities in expert calculators. In J.I.D. Campbell (Ed.) Handbook of Mathematical Cognition. Psychology Press, pp. 413-430.

Priftis, K., Zorzi. M., Meneghello. F., Marenzi. R., \& Umilta. C. (2006). Explicit versus implicit processing of representational space in neglect: Dissociations in accessing the mental number line. Journal of Cognitive Neuroscience, 18, 680-688. doi:10.1162/jocn.2006.18.4.680

Raftery, A. E. (1995). Bayesian model selection in social research. In P. V. Marsden (Ed.), Sociological methodology 1995 (pp. 111-196). Cambridge: Blackwell. 
Schneider, M., Grabner, R. H., \& Paetsch, J. (2009). Mental number line, number line estimation, and mathematical achievement: Their interrelations in grades 5 and 6. Journal of Educational Psychology, 101, 359-372. doi: 10.1037/a0013840

Shaki, S., Fischer, M. H., \& Petrusic, W. M. (2009). Reading habits for both words and numbers contribute to the SNARC effect. Psychonomic Bulletin and Review, 16, 328331. doi: 10.3758/PBR.16.2.328

Spielberger, C. D., Gorsuch, R.L., and Lushene. R.E. (1970). Manual for the StateTrait Anxiety Inventory. Palo Alto, CA: Consulting Psychologists Press.

Thayer, R. E. (1986). Activation-Deactivation Adjective Check List: Current overview and structural analysis. Psychological Reports, 58, 607-614.

van Dijck, J.-P., \& Fias, W. (2011). A working memory account for spatialnumerical associations. Cognition, 119, 114-9. doi: 10.1016/j.cognition.2010.12.013

Wood, G., Willmes, K., Nuerk, H.-C., \& Fischer, M. H. (2008). On the cognitive link between space and number: A meta-analysis of the SNARC effect. Psychology Science Quarterly, 50, 489-525. doi: 10.1027/1618-3169.52.3.187

Zorzi, M., Bonato, M., Treccani, B., Scalambrin, G., Marenzi, R. \& Priftis, K. (2012). Neglect impairs explicit processing of the mental number line. Frontiers in Human Neuroscience, 6:125. doi: 10.3389/fnhum.2012.00125 
Footnotes

1. Note that previous studies used only 20 participants (Dehaene et al., 1993;

Fischer \& Rottman, 2005).

2. Note that previous studies used only 9 or 10 repetitions per block.

3. Since those questionnaires were not relevant for the aim of the study reported here, and mostly null correlations were found, they are not reported in the main body of the paper. All results are presented in Appendix II.

4. We wish to thank the action editor Marc Brysbaert for proposing this analysis in response to power concerns raised by the reviewers. 


\section{Acknowledgements:}

We wish to thank Marc Brysbaert and two reviewers for numerous helpful comments on a previous version of the ms. In particular, we are grateful to Marc Brysbaert for putting our attention to Masson's Bayesian analysis methods, which improved the ms. a lot and might our null results "positive evidence" for a true null SNARC slope difference between skilled and unskilled participants. We also thank Franziska Burger for checking and correcting English spelling, expression and grammar. H.-C. Nuerk's research on spatial-numerical cognition was supported by the German Research Foundation (DFG) by means of a project within the Research Group (Forschergruppe) Analyse und Förderung effektiver Lehr-Lern-Prozesse (FOR 738/2/TP02). 
Table 1. Correlation of accuracy across problem subsets in Equation Verification task (upper part of the table) and correlations corrected for attenuation (lower part of the table)

\begin{tabular}{|l|c|c|c|c|}
\hline & Subset 1 & Subset 2 & Subset 3 & Subset 4 \\
\hline Subset 1 & $\mathrm{x}$ & $0.516^{*}$ & $0.563^{*}$ & $0.578^{*}$ \\
\hline Subset 2 & 0.848 & $\mathrm{x}$ & $0.496^{*}$ & $0.538^{*}$ \\
\hline Subset 3 & 0.749 & 0.837 & $\mathrm{x}$ & $0.579^{*}$ \\
\hline Subet 4 & 0.732 & 0.864 & 0.754 & $\mathrm{x}$ \\
\hline${ }^{*} \mathrm{p}<0.001$ (adjusted for multiple comparisons) \\
\hline
\end{tabular}


Table 2. Reliability of the measures used in this study:

\begin{tabular}{|l|c|}
\hline Variable & $\begin{array}{l}\text { Half-split reliability } \\
\text { (Spearman-Brown corrected) }\end{array}$ \\
\hline Simple Reaction Time & 0.991 \\
\hline Equation Verification accuracy & 0.905 \\
\hline Reaction Time in Parity Judgment task & 0.994 \\
\hline SD of Reaction Time in Simple Reaction Time & 0.981 \\
\hline SD of Reaction Time in Parity Judgment task & $\underline{0.972}$ \\
\hline Reaction Time in Equation Verification & $\underline{0.954}$ \\
\hline SD of Reaction Time in Equation Verification & $\underline{0.790}$ \\
\hline SNARC slopes & $\underline{0.698}$ \\
\hline
\end{tabular}


Table 3. Zero order correlations between SNARC slopes and continuous variables potentially predicting it.

\begin{tabular}{|l|c|}
\hline & SLOPE \\
\hline Mean RT in Parity Judgment & $-0.35^{*}$ \\
\hline SD of RT in Parity Judgment & $-0.37^{*}$ \\
\hline Simple RT & -0.04 \\
\hline SD of Simple RT & -0.03 \\
\hline Equation Verification accuracy & 0.14 \\
\hline Mean RT of Equation Verification & -0.16 \\
\hline $\begin{array}{l}\text { SD of RT of Eqation Verification } \\
\text { * p < 0.005; remain significant when adjusted for 7 } \\
\text { multiple comparisons. }\end{array}$ \\
\hline
\end{tabular}


Table 4. Results of regression analysis

\begin{tabular}{|c|c|c|c|c|c|c|}
\hline & \multicolumn{2}{|c|}{$\begin{array}{l}\text { Unstandardized } \\
\text { coefficients }\end{array}$} & \multirow{2}{*}{$\begin{array}{l}\text { Standardized } \\
\text { coefficients } \\
\text { Beta }\end{array}$} & & & \multirow{2}{*}{$\begin{array}{l}\text { Colinearity } \\
\text { VIF }\end{array}$} \\
\hline & B & $\mathrm{SE}$ & & $\mathrm{t}$ & $\mathrm{p}$ & \\
\hline Intercept & -0.04 & 10.85 & & $\begin{array}{r}<- \\
0.01\end{array}$ & 0.997 & \\
\hline $\begin{array}{l}\text { Mean RT Parity } \\
\text { Judgment }\end{array}$ & -0.04 & 0.01 & -0.40 & -2.80 & 0.007 & 1.61 \\
\hline Simple RT & 0.04 & 0.03 & 0.21 & 1.53 & 0.130 & 1.44 \\
\hline $\begin{array}{l}\text { Equations } \\
\text { Accuracy }\end{array}$ & 0.10 & 0.13 & 0.11 & 0.78 & 0.436 & 1.53 \\
\hline Equations RT & $<-0.01$ & $<0.01$ & -0.08 & -0.57 & 0.568 & 1.53 \\
\hline $\begin{array}{l}\text { Group (0= Non- } \\
\text { Math, } 1=\text { Math) }\end{array}$ & -0.57 & 2.26 & -0.35 & -0.25 & 0.800 & 1.46 \\
\hline
\end{tabular}




\section{Figure Captions:}

Figure 1: SNARC slopes and its possible correlates. The figure depicts the correlation of the SNARC slope with (i) simple RT in an independent task (top left), (ii) individual math skill in an Equation Verification task (top right), (iii) mean RT in the parity judgement task itself (bottom left), and (iv) SD RT in the Parity Judgement task itself (bottom right). SD RT was used because in other diagnostic tasks such as RT tests for attention, standard deviations are also analyzed as an alternative measure to examine whether an individual can solve the task consistently and with ease or whether influences of other representations and processes (such as magnitude) do influence the task in an inconsistent manner in some trials.

Figure 2 Proportion of participants revealing negative SNARC slopes. It can be seen that the proportion of participants showing negative SNARC slopes does not differ for the different groups. 
Figure 1

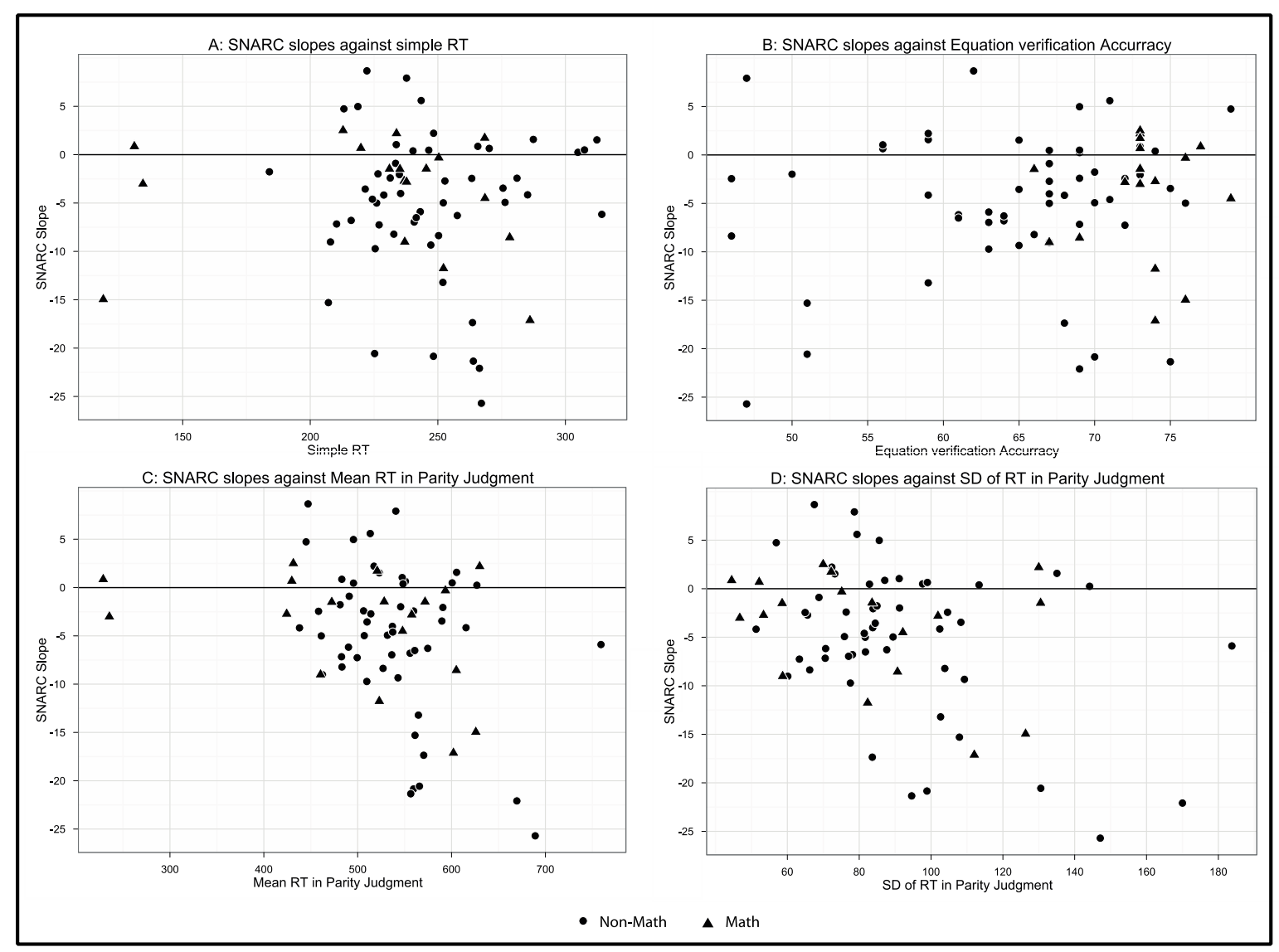


Figure 2

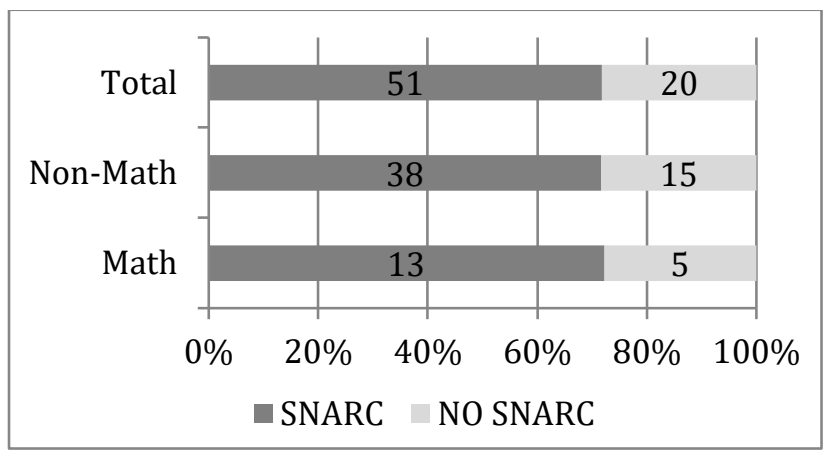


Appendix I

Material used in Equation verification task

\begin{tabular}{|c|c|c|c|}
\hline \multicolumn{2}{|c|}{$\begin{array}{l}\text { Subset 1: Addition and } \\
\text { subtraction of two-digit } \\
\text { numbers }\end{array}$} & \multicolumn{2}{|c|}{$\begin{array}{l}\text { Subset 2: One-digit by one- } \\
\text { digit/two-digit number } \\
\text { multiplication }\end{array}$} \\
\hline Correct & Incorrect & Correct & Incorrect \\
\hline $21+43=64$ & $41+34=65$ & $0 \times 15=0$ & $0 \times 12=12$ \\
\hline $25+36=61$ & $25+63=98$ & $1 \times 71=71$ & $1 \times 98=1$ \\
\hline $45+28=73$ & $54+22=86$ & $2 \times 17=34$ & $2 \times 49=99$ \\
\hline $32+57=89$ & $23+41=74$ & $3 \times 18=54$ & $3 \times 19=55$ \\
\hline $85+13=98$ & $58+23=82$ & $4 \times 23=92$ & $4 \times 23=94$ \\
\hline $73-26=47$ & $77-28=47$ & $5 \times 41=205$ & $5 \times 36=170$ \\
\hline $62-28=34$ & $64-35=28$ & $6 \times 37=222$ & $6 \times 43=248$ \\
\hline $95-13=82$ & $98-29=67$ & $7 \times 61=427$ & $7 \times 73=501$ \\
\hline $57-26=31$ & $58-27=41$ & $8 \times 24=192$ & $8 \times 17=146$ \\
\hline $15-12=3$ & $18-12=4$ & $9 \times 33=297$ & $9 \times 47=433$ \\
\hline \multicolumn{2}{|c|}{$\begin{array}{l}\text { Subset 3: Two-digit by one- } \\
\text { digit/two digit number } \\
\text { division }\end{array}$} & \multicolumn{2}{|c|}{$\begin{array}{l}\text { Subset 4: Order of operations } \\
\text { and parentheses }\end{array}$} \\
\hline Correct & Incorrect & Correct & Incorrect \\
\hline $63 / 9=7$ & $72 / 9=9$ & $3+3 \times 3=12$ & $4+4 \times 2=16$ \\
\hline $44 / 2=22$ & $66 / 3=11$ & $6-2 \times 2=2$ & $3+5 \times 8=64$ \\
\hline $36 / 6=6$ & $48 / 8=5$ & $10+9 \times 3=37$ & $2+7 \times 3=27$ \\
\hline $45 / 5=9$ & $54 / 9=7$ & $21+5 \times 4=41$ & $10+1 \times 5=55$ \\
\hline $30 / 1=30$ & $41 / 1=1$ & $50-4 \times 6=26$ & $37-1 / 6=6$ \\
\hline $54 / 27=2$ & $64 / 32=3$ & $(2+10) \times 3=36$ & $(3+6) / 3=5$ \\
\hline $64 / 16=4$ & $56 / 14=5$ & $(7+1) \times 5=40$ & $(5+6) \times 4=29$ \\
\hline $33 / 11=3$ & $55 / 11=6$ & $(9-1) \times 8=64$ & $(42+7) / 7=43$ \\
\hline $26 / 13=2$ & $38 / 19=3$ & $(12-4) / 2=4$ & $(12+13) \times 2=60$ \\
\hline $36 / 12=3$ & $42 / 14=4$ & $(20-1) \times 3=57$ & $(13-3) \times 2=7$ \\
\hline
\end{tabular}


Appendix II

Correlations between paper and pencil questionnaires and tasks reported in the study

\begin{tabular}{|l|c|c|c|c|c|}
\hline & $\begin{array}{l}\text { SNARC } \\
\text { Slope }\end{array}$ & $\begin{array}{l}\text { Mean RT } \\
\text { parity } \\
\text { judgment }\end{array}$ & $\begin{array}{l}\text { Simple } \\
\text { RT }\end{array}$ & $\begin{array}{l}\text { Equation } \\
\text { verification - RT } \\
\text { for correct trials }\end{array}$ & $\begin{array}{l}\text { Equation } \\
\text { verification } \\
\text { accuracy }\end{array}$ \\
\hline State Anxiety & 0.02 & -0.07 & -0.01 & -0.12 & $-0.26+$ \\
\hline Trait Anxiety & 0.12 & -0.15 & -0.12 & -0.01 & -0.14 \\
\hline LPT Energetic & -0.12 & 0.14 & -0.06 & 0.20 & 0.11 \\
\hline LPT Tension & -0.06 & 0.01 & 0.05 & -0.06 & -0.16 \\
\hline LPT Tiredness & 0.09 & -0.02 & 0.19 & -0.14 & -0.15 \\
\hline LPT Calmness & 0.06 & 0.00 & -0.11 & 0.03 & 0.10 \\
\hline + unadjusted p=0.031 / p adjusted for 6 multiple comparisons is 0.186 \\
\hline
\end{tabular}




\section{Appendix III}

Equation Verification task accuracy

A. Half-split reliability of each subset of the task (Spearman-Brown corrected)

\begin{tabular}{|l|r|}
\hline & Half-split reliability \\
\hline Subset 1 & 0.772 \\
\hline Subset 2 & 0.480 \\
\hline Subset 3 & 0.731 \\
\hline Subset 4 & 0.807 \\
\hline
\end{tabular}

B. Alpha Consistency for entire test and each subset

\begin{tabular}{|l|c|}
\hline & Cronbach alpha \\
\hline Entire test & 0.859 \\
\hline Subset 1 & 0.712 \\
\hline Subset 2 & 0.400 \\
\hline Subset 3 & 0.628 \\
\hline Subset 4 & 0.726 \\
\hline
\end{tabular}

C. Average mean correlation of one item with all other items of the scale for entire test and each subset

\begin{tabular}{|l|c|}
\hline & $\begin{array}{l}\text { Average correlation of one } \\
\text { item with all other items }\end{array}$ \\
\hline Entire test & 0.077 \\
\hline Subset 1 & 0.111 \\
\hline Subset 2 & 0.035 \\
\hline Subset 3 & 0.076 \\
\hline Subset 4 & 0.120 \\
\hline
\end{tabular}

D. Comparison ( $p$ values) of half-split reliabilities (Spearman-Brown corrected) of several sets using Fisher-Z transformation (unadjusted values are reported in the table, significant correlations when adjusted for multiple comparison are marked with asterisk).

\begin{tabular}{|l|l|l|l|}
\hline & Subset 2 & Subset 3 & Subset 4 \\
\hline Subset 1 & $0.003^{*}$ & 0.582 & 0.589 \\
\hline Subset 2 & & $0.017+$ & $0.001^{*}$ \\
\hline
\end{tabular}




\begin{tabular}{|l|l|l|}
\hline Subset 3 & & 0.276 \\
\hline$+\mathrm{p}<0.05$ (unadjusted) \\
${ }^{*} \mathrm{p}<0.05$ (adjusted for multiple comparisons) \\
\hline
\end{tabular}

E. Relations of SNARC slopes to Equation Verification subset scores (both accuracy and reaction times).

\begin{tabular}{|l|r|r|r|}
\hline & $\begin{array}{l}\text { Correlation } \\
\text { with } \\
\text { SNARC } \\
\text { Slope }\end{array}$ & $\begin{array}{l}\text { Bayesian likehood of } \\
\text { difference in SNARC between } \\
\text { groups (median split based } \\
\text { on given subset score; } \\
\text { p(H1|D)) }\end{array}$ & $\begin{array}{l}\text { Bayesian likehood of null } \\
\text { difference in SNARC between } \\
\text { groups (median split based } \\
\text { on given subset score; } \\
\text { p(H0|D)) }\end{array}$ \\
\hline $\begin{array}{l}\text { Subset 1 } \\
\text { ACC }\end{array}$ & -0.02 & 0.13 & 0.87 \\
\hline $\begin{array}{l}\text { Subset 2 } \\
\text { ACC }\end{array}$ & 0.09 & 0.13 & 0.87 \\
\hline $\begin{array}{l}\text { Subset 3 } \\
\text { ACC }\end{array}$ & $0.29+$ & 0.44 & 0.56 \\
\hline $\begin{array}{l}\text { Subset 4 } \\
\text { ACC }\end{array}$ & 0.12 & 0.11 & 0.89 \\
\hline $\begin{array}{l}\text { Subset 1 } \\
\text { RT }\end{array}$ & -0.20 & 0.52 & 0.48 \\
\hline $\begin{array}{l}\text { Subset 2 } \\
\text { RT }\end{array}$ & -0.01 & 0.11 & 0.73 \\
\hline $\begin{array}{l}\text { Subset 3 } \\
\text { RT }\end{array}$ & -0.19 & 0.27 & 0.80 \\
\hline $\begin{array}{l}\text { Subset 4 } \\
\text { RT }\end{array}$ & -0.16 & 0.20 & \\
\hline +p=0.014 (unadjusted for 8 multiple comparisons), padj=0.12 & \\
\hline
\end{tabular}

F. Comparison of correlation coefficients ( $p$ values) of given subset score and SNARC slopes.

\begin{tabular}{|l|c|c|c|}
\hline & Subset 2 & Subset 3 & Subset 4 \\
\hline Subset 1 & 0.522 & 0.063 & 0.412 \\
\hline Subset 2 & & 0.226 & 0.857 \\
\hline Subset 3 & & & 0.298 \\
\hline
\end{tabular}

